\title{
Efficient synthesis of interfacially polymerized membranes for solvent resistant nanofiltration
}

\author{
Sanne Hermans ${ }^{\mathrm{a}}$, Elke Dom ${ }^{\mathrm{a}}$, Hanne Mariën ${ }^{\mathrm{a}}$, Guy Koeckelberghs ${ }^{\mathrm{b}}$, Ivo F.J. Vankelecom ${ }^{\mathrm{a}}{ }^{*}$ \\ ${ }^{\text {a }}$ Centre for Surface Chemistry and Catalysis, KU Leuven, Kasteelpark Arenberg 23, PO Box 2461, \\ 3001 Leuven, Belgium; ${ }^{b}$ Polymer Chemistry and Materials, KU Leuven, Celestijnenlaan 200F, PO \\ Box 2404, 3001 Leuven, Belgium \\ *Corresponding author: ivo.vankelecom@biw.kuleuven.be, tel: +3216321594
}

\begin{abstract}
Thin film composite (TFC) membranes are used worldwide in aqueous applications. They mostly consist of a polyamide top-layer put on a polysulfone support via interfacial polymerization. Due to their thin and dense selective layer, these membranes are also interesting for filtrations in organic solutions. Polysulfone should then be replaced by a more solvent resistant material. The synthesis of solvent resistant nanofiltration TFC membranes via a newly developed method is reported: phase inversion, crosslinking and impregnation of a polyimide support are combined by adding amines to the aqueous coagulation bath. Next, a thin polyamide top-layer is formed on the support via interfacial polymerization. Several amines are tested as crosslinker for the support and as monomer for top-layer formation. The use of an amine mixture is also explored. Membrane stability, time in the coagulation bath, effect of solvent activation and mass \& solvent intensity of the process are investigated. This novel synthesis method minimizes the use of (hazardous) materials, thus requires less reagents and creates less waste. Moreover, time and effort are saved during the synthesis process, which is of great interest for membrane producers and from an environmental point of view.
\end{abstract}

\section{KEYWORDS}

Thin film composite; Interfacial polymerization; Solvent resistant Nanofiltration; Membrane Synthesis; Polyimide crosslinking 


\section{INTRODUCTION}

Thin film composite (TFC) membranes synthesized via interfacial polymerization are abundantly used in aqueous applications, e.g. desalination. These membranes consist of a very thin (50 to $500 \mathrm{~nm}$ ), yet dense top-layer, allowing high water permeances, while small solutes (like salts) are effectively retained [1]. Because of this ultrathin and crosslinked selective layer, such TFC membranes could be particularly interesting to improve the performance of current solvent resistant nanofiltration (SRNF) membranes. In SRNF, separations down to the molecular level can be realized in solvent streams by simply applying an external pressure over a membrane. It is an emerging technique in the food, pharmaceutical and (petro)chemical industry [2-4]. Today, commercially available SRNF membranes are mostly integrally skinned asymmetric membranes, having rather low solvent permeances, due to their intrinsically thicker selective layer (hence more resistance to mass transfer through the membrane) [5].

To prepare TFC membranes for aqueous applications, a porous polysulfone (PSf) support layer is typically impregnated with an aqueous diamine solution and subsequently put in a hexane-based acyl chloride solution. At the interface of both immiscible solvents, the monomers react, forming a thin, dense polyamide (PA) top-layer on the support [6]. Previously, we reported a method to combine phase inversion of the PSf support and its impregnation with amine monomers by using an aqueous amine solution as the coagulation bath [7]. For applications in many industrially relevant organic solvents (e.g. dimethylformamide, DMF), the typically used PSf support must be replaced by a more solvent resistant material, since PSf is known to swell extensively (or even dissolve) in many of these solvents. A well-known solvent resistant material for membrane applications is crosslinked polyimide (PI) [8, 9]. Recently, this material was used as a support for the synthesis of SRNF TFC membranes and showed promising results [10, 11]. However, the synthesis process of these membranes was cumbersome, time-consuming and required the use of a conditioning agent (polyethylene glycol) and large amounts of solvent (isopropanol, IPA) at different stages. Firstly, the PI dope solution was turned into a solid support membrane via phase inversion in a coagulation bath containing pure water. The formed support was kept in an IPA solvent exchange bath to remove residual water and preparation solvents. Subsequently, it was crosslinked by immersion in a solution of hexanediamine (HDA) in IPA for $16 \mathrm{~h}$ and washed with IPA for $3 \mathrm{~h}$ to remove residual HDA. IPA is used as 
the solvent for crosslinking because the PI support will swell in this medium, making the polymer chains thus more available for crosslinking by the diamine. After the crosslinking procedure, the support still had to be conditioned (in polyethylene glycol/IPA) overnight, before it could be dried and finally impregnated with the first monomer for the top-layer formation.

Vanherck et al. developed a simplified procedure for the crosslinking of PI membranes [12]. Herein, phase inversion and crosslinking are combined, by adding the diamine crosslinker directly to the water coagulation bath in which the phase inversion takes place, instead of crosslinking the membrane in a separate step by immersion in a diamine/organic solvent mixture. The crosslinking reaction thus occurs simultaneously with the membrane formation in the simplified procedure, avoiding the use of organic solvent and increasing efficiency, since the diamine no longer has to diffuse into the already solid membrane matrix for reaction to take place. The membranes prepared by Vanherck et al. were integrally skinned asymmetric membranes (thus without an extra top-layer) and directly applicable in SRNF. The thickness of their selective layer however limits the possibility to achieve high solvent permeances. In principle, the use of a thin selective layer, as in TFC membranes, would be more efficient as higher permeances through the selective layer can then be obtained.

In this work, a new synthesis process was developed to prepare SRNF TFC membranes with PA top-layers on crosslinked PI supports. As mentioned previously, in TFC membrane synthesis via interfacial polymerization, the support is commonly impregnated with an aqueous diamine solution (in a separate step). In this newly developed method, diamines are added to the coagulation bath to act simultaneously as both a crosslinker for the PI support and a monomer for the top-layer formation. This way, three steps are now carried out simultaneously (further called the SIM process): phase inversion, crosslinking and monomer impregnation of the support. Moreover, the SIM process only requires the use of water as coagulation/reaction medium. In addition, the conditioning step is avoided, since drying of the support in between processing steps is no longer required.

A schematic overview of the traditional versus the SIM process for the synthesis of SRNF TFC membranes is presented in Scheme 1. This new method thus drastically shortens processing time, but also prevents the use of chemicals, i.e. polyethylene glycol and substantial quantities of IPA at different stages. Hence, it requires less reagents and creates 
less waste during the membrane synthesis. Moreover, the traditional problem of rewetting every single surface pore of the support with the first monomer solution (as a major cause of pinhole creation in the final TFC membrane) is avoided here since this monomer is intrinsically present already in each pore. Indeed, pores originate where non-solvent has nucleated and this non-solvent readily contains the reagent.

Scheme 1. Comparison of traditional and SIM process for synthesis of SRNF TFC membranes

\section{Traditional process}

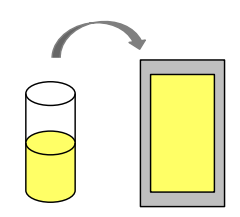

Polymer Cast solution film

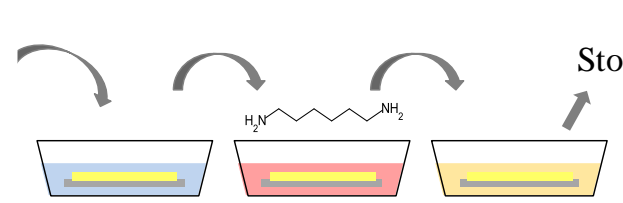

Phase inversion
Crosslinking Conditioning

Impregnation monomer 1

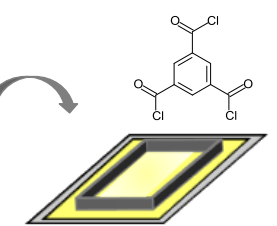

Interfacial

Polymerization monomer 2

\section{SIM process}

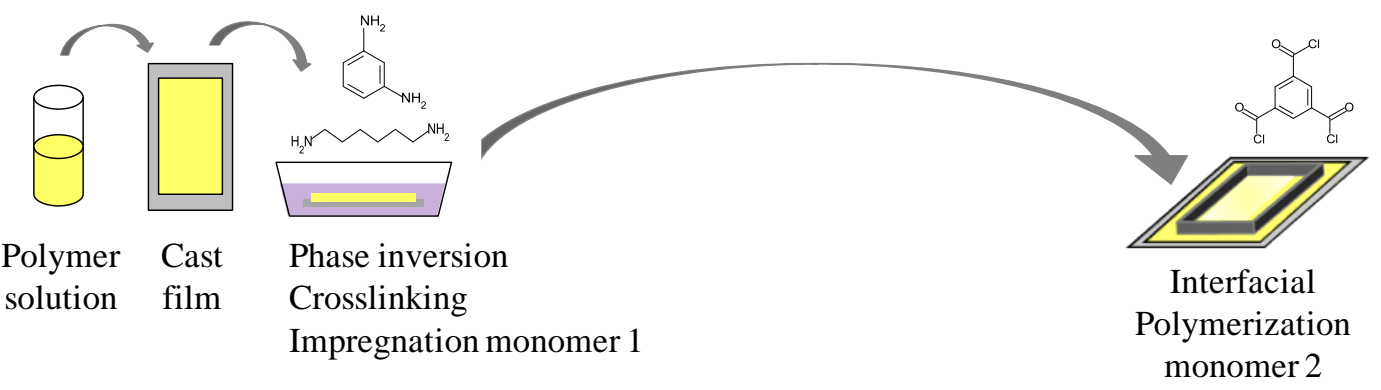

\section{EXPERIMENTAL}

\subsection{Materials}

Matrimid $^{\circledR} 9725$ PI was obtained from Huntsman (Switzerland). The non-woven polypropylene/polyethylene (PP/PE) fabric Novatexx 2471 was kindly provided by Freudenberg (Germany). Propanediamine (PDA, Chimica, 99\%), butanediamine (BDA, Acros, 99\%), hexanediamine (HDA, Acros, 99.5\%), octanediamine (ODA, Fluka, 98\%), tris(2-aminoethyl)amine (polyamine, Sigma Aldrich, 98\%+) and meta-phenylenediamine (MPD, Acros, 99+\%) were applied as amines. Their chemical structures are shown in Figure 1. Trimesoyl chloride (TMC, Acros, 98\%), N,N-dimethylformamide (DMF, Sigma Aldrich, 99+\%), dimethyl sulfoxide (Acros, 99.5\%), N-methyl-2-pyrrolidone (NMP, Acros, 99\%), 
tetrahydrofuran (THF, Chemlab, 99.9\%), methanol (MeOH, VWR, 99.5+\%), ethanol (EtOH, VWR, 99.8+\%) and hexane (VWR, 97+\%) were used as received. Rose Bengal (RB, Fluka) was applied as test solute. All experiments were carried out with Milli-Q water.

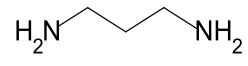

PDA

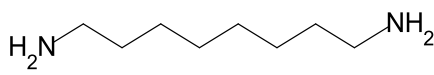

ODA

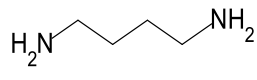

BDA

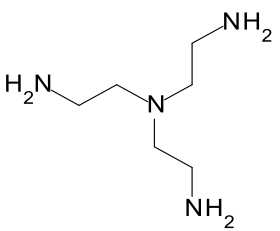

polyamine<smiles>NCCCCCCN</smiles>

HDA

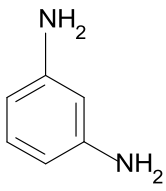

MPD

Figure 1. Chemical structures of the amines used in the coagulation bath.

\subsection{Membrane synthesis}

\subsubsection{PI support}

Support membranes were synthesized via phase inversion. A homogeneous solution of 14 wt $\%$ Matrimid PI (dried overnight at $110^{\circ} \mathrm{C}$ ) in $\mathrm{NMP} / \mathrm{THF}(3 / 1)$ was cast on a polypropylene/polyethylene non-woven (Novatexx 2471, Freudenberg), which was first impregnated with NMP, at constant speed $(77 \mathrm{~mm} / \mathrm{s})$ using an automatic casting device (Braive Instruments, Belgium) at $200 \mu \mathrm{m}$ wet thickness. The cast polymer film was then immersed in an aqueous amine solution of $0.185 \mathrm{M}$, unless specified otherwise. The coagulation time was $30 \mathrm{~min}$, unless specified otherwise.

\subsubsection{Thin film composite}

A thin PA layer was synthesized on top of the support via interfacial polymerization. The excess aqueous solution was removed from the PI support using a rubbery wiper. A solution of $0.1 \%(\mathrm{w} / \mathrm{v}) \mathrm{TMC}$ in hexane was subsequently poured gently on the impregnated support layer. The hexane solution was drained off after $60 \mathrm{~s}$ of polymerization and the membrane was rinsed with hexane to remove unreacted TMC. After 1 min air drying, the membrane was put in a water bath to remove unreacted amine. Finally, the synthesized TFC membrane was stored in distilled water. Some TFC membranes were treated with an activating solvent. This was done by storing them in DMF or DMSO for a certain period prior to filtration. 


\subsection{Membrane characterization}

\subsubsection{Filtration experiments}

The membrane performance was tested in a high throughput filtration module with an EtOH or $\mathrm{MeOH}$ feed and the dye RB $(1017 \mathrm{Da}, 35 \mu \mathrm{M})$ as a test solute for SRNF $[13,14]$. The filtration module allowed six simultaneous dead-end filtrations under the exact same operating conditions. The active area of each membrane inside the module was $5.31 \cdot 10^{-4} \mathrm{~m}^{2}$. A nitrogen pressure of 1 bar was applied for the support membranes and 20 bar for the TFC membranes. The feed was stirred rigorously at $400 \mathrm{rpm}$ to minimize concentration polarization. At least three coupons of each membrane sheet were tested.

Membrane permeance $\left(\mathrm{L}_{\mathrm{p}}\right)$ was calculated using:

$$
\mathrm{L}_{\mathrm{p}}=\frac{\mathrm{V}}{\mathrm{A} \cdot \mathrm{t} \cdot \Delta \mathrm{P}}
$$

where, $\mathrm{V}$ is the permeate volume $(\mathrm{L}), \mathrm{A}$ is the membrane area $\left(\mathrm{m}^{2}\right), \mathrm{t}$ is the time $(\mathrm{h})$ and $\Delta \mathrm{P}$ is the applied pressure (bar).

The $\mathrm{RB}$ retention was calculated using:

$$
\mathrm{R}=\frac{\mathrm{c}_{\mathrm{f}}-\mathrm{c}_{\mathrm{p}}}{\mathrm{c}_{\mathrm{f}}} \cdot 100
$$

in which $c_{f}$ and $c_{p}$ are the feed and the permeate concentration, respectively. Solute concentrations were measured on a Perkin-Elmer lambda $12 \mathrm{UV}$-Vis spectrophotometer (550 $\mathrm{nm}$ in $\mathrm{EtOH}, 552 \mathrm{~nm}$ in $\mathrm{MeOH})$.

\subsubsection{Electron microscopy}

The cross-section morphology of the support was analyzed with scanning electron microscopy (SEM), on a Philips XL 30 FEG SEM. Samples were broken in liquid nitrogen and coated with a thin $(1.5-2 \mathrm{~nm})$ gold layer using a Cressington HR208 high resolution sputter coater.

Transmission electron microscopy (TEM) was applied for top-layer visualization at higher resolution. Unstained membrane samples were embedded in an araldite resin (Polyscience) and cut into ultrathin $(70 \mathrm{~nm})$ cross-sections with a Reichert Ultracut E microtome. Images 
were taken with a Zeiss EM900 TEM. Top-layer thickness was calculated as the average of 20 equidistant spots along the entire cross-section.

\subsubsection{Attenuated total reflectance infrared (ATR-IR) spectroscopy}

The chemical composition of the membrane surface was analyzed by ATR-FTIR. Spectra were collected from dried membranes using a Bruker ALPHA-P FT-IR spectrometer with a diamond ATR crystal. Thirty-two scans were collected at a resolution of $4 \mathrm{~cm}^{-1}$ and for every membrane at least three different positions were measured.

\subsubsection{Mass and solvent intensity}

The mass and solvent intensities of a TFC membrane synthesis method were calculated according to the definitions presented by Jimenez-Gonzalez et al. [15]:

$$
\begin{aligned}
& \text { Mass intensity }=\frac{\text { mass of all materials used excluding water }}{\text { mass of product }} \mathrm{kg} / \mathrm{kg} \text { product } \\
& \text { Solvent intensity }=\frac{\text { mass of all solvent used excluding water }}{\text { mass of product }} \mathrm{kg} / \mathrm{kg} \text { product }
\end{aligned}
$$

To allow a better comparison between the values of the traditional and the SIM process, the mass of water was included in some of the calculations (further specified in the text).

\subsubsection{Leachable fraction}

To determine their leachable fraction, pieces of PI support were dried and weighed $\left(\mathrm{m}_{\mathrm{i}}\right)$. Subsequently, the pieces were soaked in DMF for $24 \mathrm{~h}$, dried in a vacuum oven and weighed again $\left(\mathrm{m}_{\mathrm{f}}\right)$. Their leachable fraction was determined based on the following equation, in which $\mathrm{m}_{\mathrm{i}}$ and $\mathrm{m}_{\mathrm{f}}$ are the initial and final mass of the support, respectively:

$$
\text { Leachable fraction }(\%)=\frac{\mathrm{m}_{\mathrm{i}}-\mathrm{m}_{\mathrm{f}}}{\mathrm{m}_{\mathrm{i}}} \cdot 100
$$




\section{RESULTS AND DISCUSSION}

\subsection{Crosslinking of the PI support}

Using MPD, the most common monomer for interfacial polymerization, as amine in the coagulation bath, PI supports could be prepared following the novel synthesis method, but they readily dissolved in DMF. It is indeed known that sterical hinder causes MPD to be a less efficient crosslinker for PI [16].

In order to find a suitable amine that can act as both a crosslinker for the support and as a monomer for the top-layer formation, a series of amines was tested. p-Xylylenediamine (XDA) and hexanediamine (HDA) are adequate crosslinkers for PI, but the limited solubility of XDA in water makes it an unfit candidate for the SIM process, in which water is an essential element for phase inversion of the support $[12,17,18]$. As a preliminary test, the polymerization reaction of various known PI crosslinkers - PDA, BDA, HDA, ODA and polyamine (Figure 1) - was studied by simply contacting an aqueous amine solution with a hexane-based TMC solution $[19,20]$. For each amine, a turbid structure formed within a few seconds at the interface between the liquids, confirming PA formation.

To unravel the dual role of the amines in the coagulation bath (as crosslinkers for the PI support matrix and as monomers for the PA top-layer formation) crosslinked PI support membranes were first prepared to study the effect of the amine presence in the coagulation bath on the support properties as such. Each time, an aqueous amine solution of $0.185 \mathrm{M}$ (equivalent to $2 \mathrm{wt} \% \mathrm{MPD}$ ) was used. All prepared supports were resistant to DMF, giving a first indication of successful crosslinking.

To study the chemical changes induced by crosslinking of the support, ATR-FTIR spectra were measured (Figure 2). The nucleophilic attack of an amine group on a carbonyl group of an imide, creates two amide groups [9]. A clear intensity decrease in imide bands and increase in amide bands was observed for the supports formed in the presence of amines, confirming successful crosslinking. 


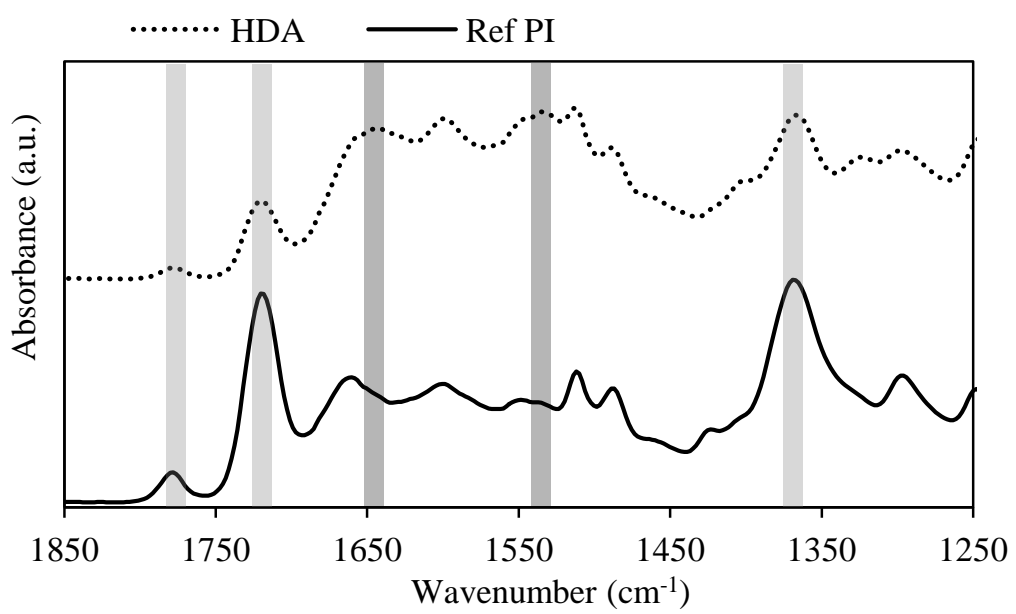

Figure 2. ATR-FTIR spectra of PI support membranes coagulated in pure water (Ref PI, full line) and in an aqueous solution of $0.185 \mathrm{M}$ HDA (HDA, dotted line). Imide bands (marked light grey) are observable at $1780 \mathrm{~cm}^{-1}(\mathrm{C}=\mathrm{O}), 1718 \mathrm{~cm}^{-1}(\mathrm{C}=\mathrm{O})$ and $1363 \mathrm{~cm}^{-1}(\mathrm{C}-\mathrm{N})$, amide bands (marked dark grey) at $1645 \mathrm{~cm}^{-1}(\mathrm{C}=\mathrm{O})$ and $1534 \mathrm{~cm}^{-1}(\mathrm{C}-\mathrm{N})$.

The cross-section morphology of the membranes prepared via the SIM process (with each time a different type of amine in the coagulation bath) was studied with SEM. Figure 3 illustrates the highly similar appearance of the supports: all show fingerlike macrovoids with a denser skin just above. These images already suggest that the role of the amine type in the coagulation bath does not have a large influence on the phase inversion process of the support. A piece of the thin PA top-layer can also be seen on Figure 3A, partly falling over the cross-section.
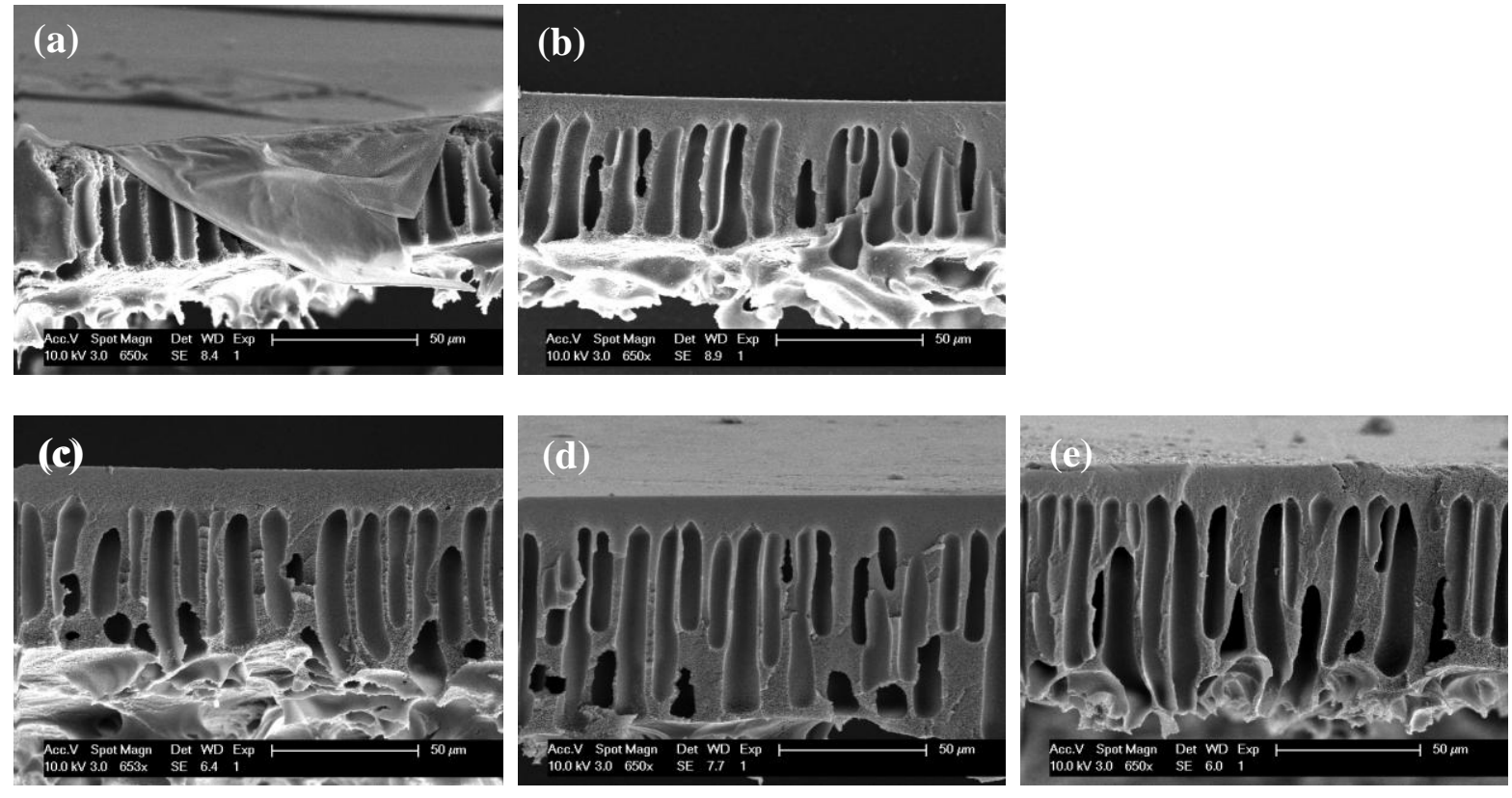
Figure 3. Cross-section SEM images of TFC membranes prepared via the SIM process with various amines in the coagulation bath: (a) PDA, (b) BDA, (c) HDA, (d) ODA and (e) polyamine.

The performance of the crosslinked supports was tested in a high throughput filtration module with an EtOH feed and the dye RB (1017 Da) as a test solute for SRNF [13, 14].

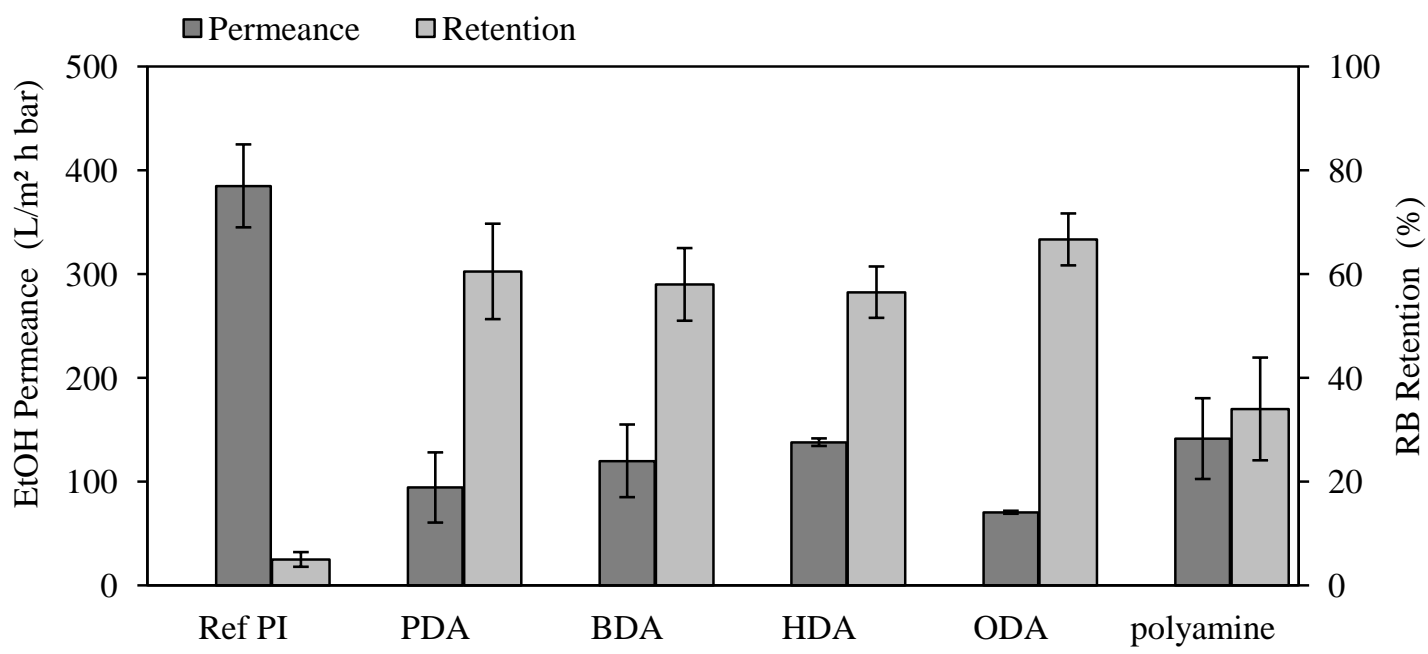

Figure 4. Permeance and retention of PI support membranes prepared via the SIM process with various amine solutions. An uncrosslinked PI support coagulated in pure water is added as a reference $(\operatorname{Ref} \mathrm{PI})$.

A significant decrease in EtOH permeance and increase in $\mathrm{RB}$ retention was observed when replacing the pure water coagulation medium (Ref PI) by an amine solution (Figure 4). This suggests an active role of the amines in the phase inversion process, where they can be considered as non-solvent additives [21].

Using polyamine resulted in a slightly less selective support which might be due to the less efficient crosslinking caused by steric hindrance of this amine and the amine's hydrophilic nature, impeding sorption in the rather hydrophobic PI matrix [22]. The octanol-water partition coefficient is the ratio of concentrations of a compound in the two phases of an octanol-water mixture at equilibrium. It is thus a measure for a compound's hydrophobicity. The $\log \mathrm{K}_{\mathrm{ow}}$ value of the polyamine is -2.90 , while HDA has a $\log \mathrm{K}_{\mathrm{ow}}$ of 0.22 . In addition, its role in the membrane solidification process during phase inversion might also be different due to the above mentioned properties. Since the crosslinking effect of the various amines on the support is thus quite comparable (see also similar morphologies in Figure 3), it can be 
assumed that possible differences in the TFC performance will be determined by variations in the PA top-layer rather than by differences in the supports.

\subsection{Synthesis of SRNF TFC membranes using various amines}

Next, TFC membranes were prepared via the SIM process as described in the experimental section. All resulting TFC membranes were resistant to DMF. Comparing their performance (Figure 5) to that of the corresponding support membranes (Figure 4), confirmed the formation of a dense, selective PA top-layer: higher RB retentions were observed and EtOH permeance decreased three orders of magnitude.

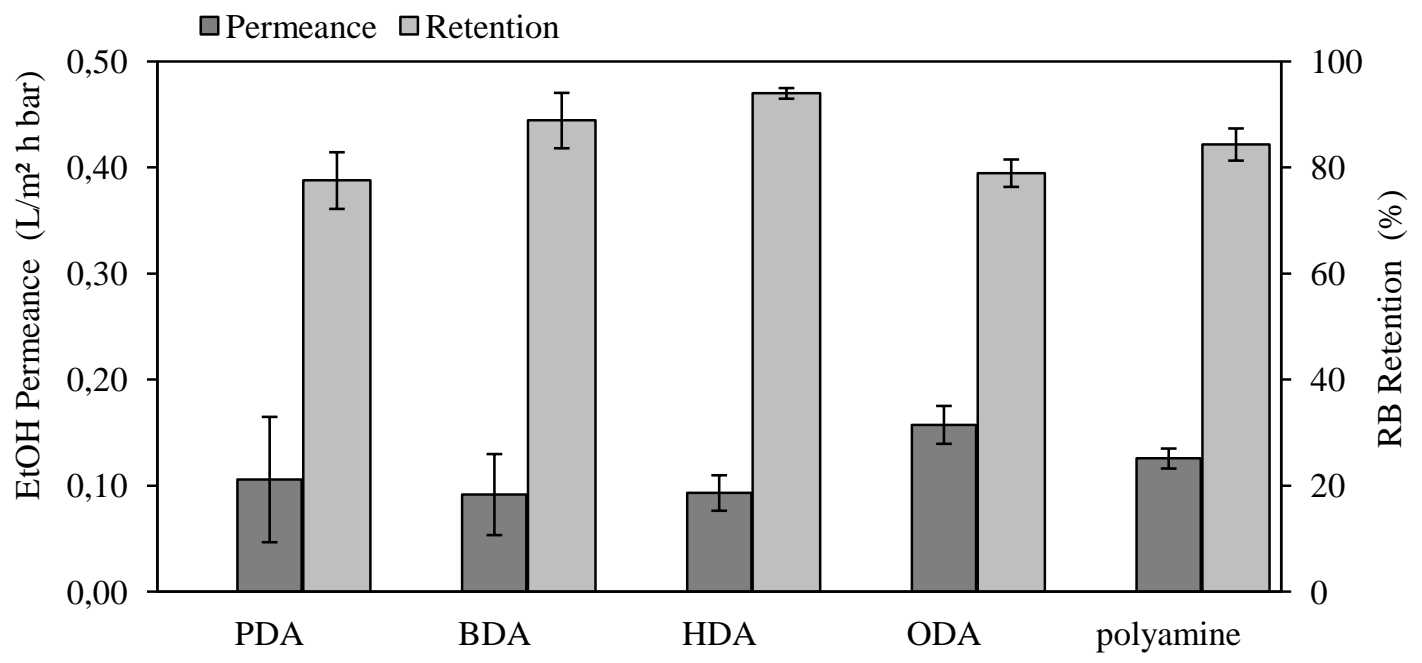

Figure 5. Permeance and retention of TFC membranes prepared via the SIM process with various amine solutions as coagulation baths.

Considering both permeance and retention of the TFC membranes in Figure 5, HDA appears to be the optimal amine choice. Perhaps, its chain flexibility makes it well suited to react twice and form a dense PA network [23]. Using PDA resulted in a TFC membrane with low retention. Possibly, the slightly lower nucleophilic nature of the shorter PDA molecules affects their reaction with the acyl chloride, resulting in a less dense PA network. Also, PDA is more hydrophilic compared to HDA, which will influence its diffusivity and solubility in the organic phase, hence, affect the PA top-layer formation [24]. When ODA was used as a crosslinker and monomer for top-layer formation, the resulting TFC membrane showed a higher permeance and lower retention than when using HDA. Diamines with longer aliphatic chains increase the free volume in the PA top-layer [25]. An increase in chain length of the aliphatic crosslinker, increases the interchain distance and thus creates a less compact 
membrane structure, resulting in a higher permeance and lower retention. The TFC membrane prepared with polyamine shows a slightly higher permeance and lower retention compared to HDA, presumably due to its larger size and multifunctionality. The interfacial polymerization (and crosslinking of the support) are done randomly, creating a disordered structure, preventing the polymer chains from getting properly organized and creating a defect-free network. It is possible that the large size of the polyamine hinders its diffusion through the forming PA barrier towards the organic phase, thus, impeding the formation of a uniform, defect-free PA top-layer.

\subsection{Synthesis of SRNF TFC membranes from an HDA/MPD mixture}

The previous results prove that it is possible to prepare TFC membranes via the SIM process, but the performance of the prepared membranes is still rather low. Using other monomers results in different PA structures, manifesting itself in different membrane performances. HDA is a well-known crosslinker for PI, but given the low permeance of the TFC membrane, its performance as a monomer for top-layer formation via the SIM process can probably still be optimized. Previously, others used HDA as a monomer for PA synthesis via interfacial polymerization and showed that TFC membranes prepared from aliphatic diamines show an inferior performance in aqueous applications compared to membranes prepared from aromatic diamines (like MPD) [26]. Membranes consisting of a fully aromatic network show higher rupture strengths, higher salt retentions and comparatively higher water fluxes. Also, it was shown that MPD based membranes have a larger free volume than HDA based membranes [25].

\subsubsection{Crosslinker concentration}

Previous research indicated that $2.0 \mathrm{wt} \% \mathrm{MPD}$ is adequate for the formation of a wellperforming PA top-layer [7]. To minimize the amount of reagents needed for membrane synthesis, the required crosslinker concentration to obtain a crosslinked support was first determined. The HDA concentration in the coagulation bath was varied from $0.10 \mathrm{wt} \%$ to $1.00 \mathrm{wt} \%$ and the leachable fraction of the pure supports was measured. The results in Table 1 illustrate that after $30 \mathrm{~min}$ of immersion in $0.50 \mathrm{wt} \% \mathrm{HDA}$, a leachable fraction of only $0.16 \%$ in DMF was obtained, in agreement with Vanherck et al. [12]. Therefore, a mixture of 
$0.5 \mathrm{wt} \% \mathrm{HDA}$ and $2.0 \mathrm{wt} \% \mathrm{MPD}$ was used in the coagulation bath for further synthesis of SRNF TFC membranes.

Table 1. Leachable fraction of PI supports crosslinked in coagulation baths with varying HDA concentrations.

\begin{tabular}{cc}
\hline $\begin{array}{c}\text { HDA concentration } \\
(\mathrm{wt} \%)\end{array}$ & $\begin{array}{c}\text { Leachable fraction } \\
(\%)\end{array}$ \\
\hline 0.10 & 1.28 \\
0.25 & 0.56 \\
0.50 & 0.16 \\
0.75 & 0.16 \\
1.00 & 0.10 \\
\hline
\end{tabular}

\subsubsection{Coagulation bath containing HDA and MPD}

Using an amine mixture of $0.5 \mathrm{wt} \% \mathrm{HDA}$ and $2.0 \mathrm{wt} \%$ MPD in the coagulation bath resulted in TFC membranes with an improved performance: the permeance increased from $0.09 \mathrm{~L} / \mathrm{m}^{2}$ $\mathrm{h}$ bar, when using only HDA, to $0.17 \mathrm{~L} / \mathrm{m}^{2} \mathrm{~h}$ bar when using the mixture. Meanwhile, the retention of RB increased from $94.0 \%$ to $97.5 \%$. It was already mentioned earlier that MPD alone did not sufficiently crosslink the PI support, likely due to sterical hinder. Hence, MPD as such can not be used to prepare SRNF TFC membranes via the SIM method as the PI support would simply dissolve in aggressive solvents. Since HDA is more reactive towards PI (and added in a lower concentration), it will act mainly as the support crosslinker here, while MPD ensures the formation of a proper selective PA top-layer. Nevertheless, the incorporation of small amounts of HDA in the top-layer is possible. The binary amine mixture thus has a diversified role in the membrane formation during the SIM process. TEM images of the support and TFC membrane cross-sections showed the typical ridge and valley structure of an aromatic PA top-layer, with an active layer thickness of $93 \pm 18 \mathrm{~nm}$ (Figure $6)$. 

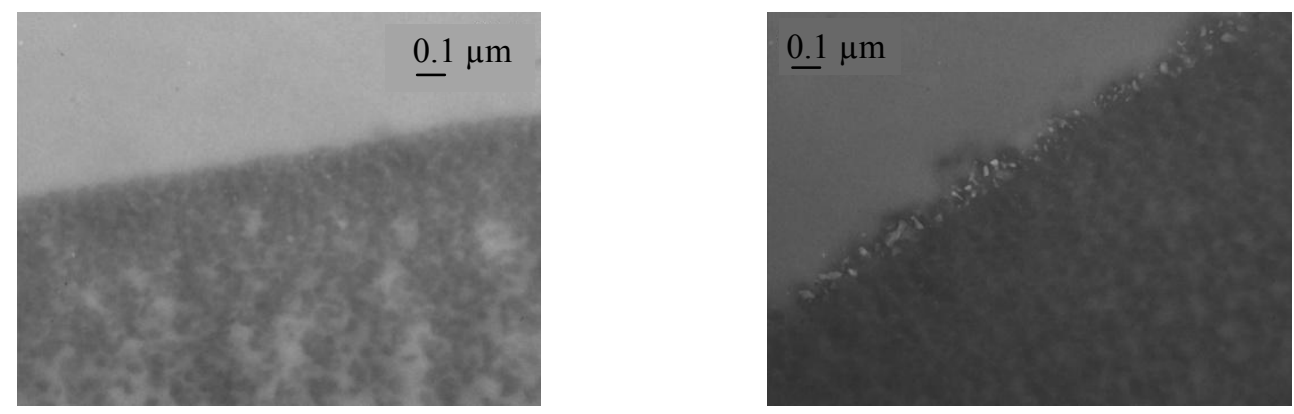

Figure 6. Cross-section TEM images of a PI support (left) and a TFC membrane (right) prepared via the SIM process. The aqueous coagulation bath contained $0.5 \mathrm{wt} \% \mathrm{HDA}$ and 2.0 wt $\%$ MPD.

To still further increase the performance, the TFC membranes were treated with DMF or DMSO as activating solvents prior to filtration. It was proven earlier that this post-treatment irreversibly improves the permeance of SRNF TFC membranes without compromising retention $[10,11]$. DMF and DMSO were chosen because their Hildebrand solubility parameters (24.9 $\mathrm{MPa}^{0.5}$ and $26.6 \mathrm{MPa}^{0.5}$ respectively) are similar to the one of aromatic PA $\left(23.0 \mathrm{MPa}^{0.5}\right)$, i.e. they are good swelling agents for PA [27]. Solvent activation is believed to cause a temporary swelling of the top-layer, so that PA oligomers will dissolve in the activating solvent. By swelling of the PA layer on exposure to the activating solvent, additional free volume (previously blocked by these low molecular weight PA fragments) would thus be created in the top-layer with a higher permeance as a result [10, 28]. A similar permeance increasing effect has been reported after treating TFC membranes with EtOH and IPA, and was attributed to the removal of small molecular fragments because of partial PA dissolution in the alcohols [29]. The effect of treatment with DMF or DMSO is much stronger, likely due to their excellent solvent power for the PA fragments. The (limited) dissolution of PA fragments from the top-layer would logically go together with a decrease in selectivity. However, increased retentions are observed after solvent treatment. It was suggested that imperfections or defects in the top-layer are removed because of annealing effects during the swelling operation, resulting in an increased retention [29]. The dissolution of small PA fragments together with this process of "surface defect healing" is believed to actually increase both permeance and retention [30,31]. Although this is an acceptable hypothesis, the 'solvent activation effect' is surely not yet fully elucidated.

As can be seen in Figure 7, a 12h storage of the SRNF TFC membrane in DMF prior to filtration already improved the performance dramatically: permeance increased by a factor 
5.5 and RB passage (i.e., 100-Retention) decreased with 50\%. Storing the membranes for $24 \mathrm{~h}$ in DMF led to an even better performance: permeance increased by a factor 16 and RB was $100 \%$ retained. Since DMF is an environmentally harmful solvent, the effect of a green activating solvent, i.e. DMSO, was also investigated. Similar trends were observed: storing the membranes for $12 \mathrm{~h}$ in DMSO increased the permeance by a factor 3.5 and decreased the RB passage with $60 \%$. Storage for $24 \mathrm{~h}$ in DMSO increased the permeance by a factor 5.5 and decreased the RB passage with $88 \%$. Although the activating effect of the environmentally friendly DMSO seems to be less strong, its use is preferred over the use of toxic DMF [18].

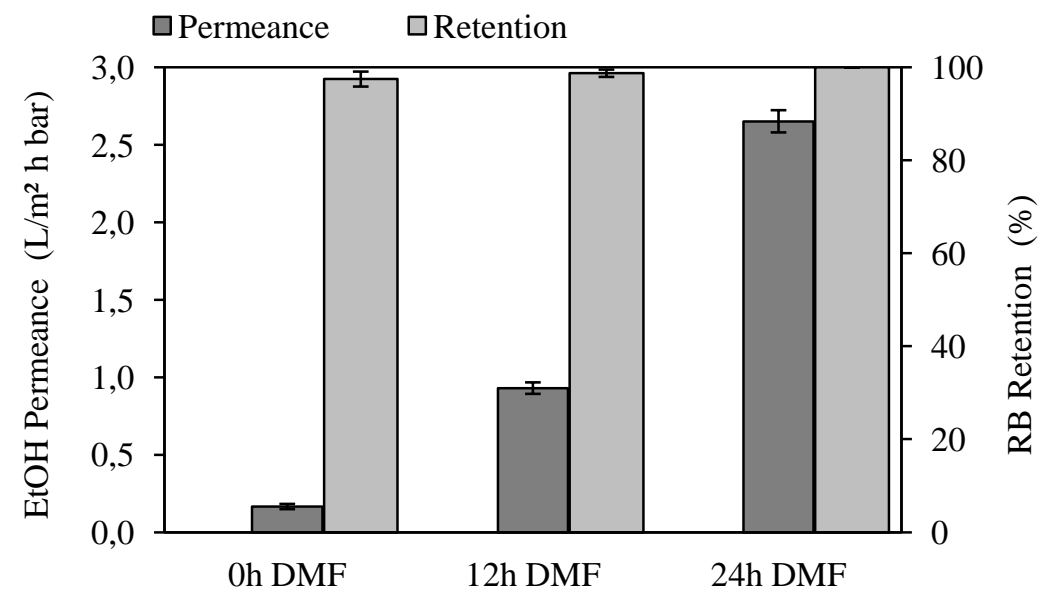

Figure 7. Effect of activating solvent prior to filtration on the performance of SRNF TFC membranes, synthesized via the SIM process with a mixture of HDA and MPD in the coagulation bath.

\subsection{Time in the coagulation bath}

Previous work showed that 5 min of immersion in the coagulation bath was by far sufficient to complete the phase inversion process and ensure a good impregnation of a PSf support with amine (MPD) [7]. Using this new SIM method, crosslinking of the PI support occurs also in the coagulation bath, simultaneously with the phase inversion process and the impregnation of the support with MPD. Therefore, 30 min of immersion were applied until now, which resulted in membranes with a good stability. To investigate whether shorter immersion times were possible, the time in the coagulation bath was varied between 5 and 30 min. Subsequently, the leachable fraction of the synthesized supports was studied. 
Table 2. Leachable fraction (after a $72 \mathrm{~h}$ storage in DMF) of a PI support prepared with varying immersion times in the coagulation bath containing $2.0 \mathrm{wt} \%$ MPD and $0.5 \mathrm{wt} \%$ HDA in milliQ water.

\begin{tabular}{cc}
\hline $\begin{array}{c}\text { Time in } \\
\text { coagulation bath } \\
(\mathrm{min})\end{array}$ & $\begin{array}{c}\text { Leachable } \\
\text { fraction } \\
(\%)\end{array}$ \\
\hline 5 & 2.01 \\
10 & 0.53 \\
15 & 0.44 \\
20 & 0.41 \\
25 & 0.38 \\
30 & 0.21 \\
\hline
\end{tabular}

The leachable fraction of a PI support after storage in DMF is presented in Table 2. The prepared supports were already thoroughly crosslinked after $5 \mathrm{~min}$ of immersion. The leachable fraction of the support that was immersed in the coagulation bath for 10 min was only $0.53 \%$. Prolonging the immersion time in the coagulation bath resulted in even lower leachable fractions. To successfully synthesize a top-layer, a good uptake of monomer in the support is also important. Generally, a support is immersed in aqueous amine solutions for several minutes $[7,10]$. Since the amines are already present in the coagulation bath when applying the SIM method for the synthesis of SRNF TFC membranes, amine impregnation occurs while the solid support matrix is formed, guaranteeing an instantaneous monomer uptake in its pores. Thus, $10 \mathrm{~min}$ of immersion in a coagulation bath containing $2.0 \mathrm{wt} \%$ MPD and $0.5 \mathrm{wt} \%$ HDA is certainly sufficient to complete the phase inversion process, crosslinking and monomer impregnation of the PI support. The required time is thus even shorter than the 30 min that were applied in the previous experiments.

\subsection{Comparison with the traditional TFC synthesis process}

To compare the performance of the SIM membranes to that of membranes prepared via the traditional process of interfacial polymerization, filtrations were done with a $\mathrm{MeOH} / \mathrm{RB}$ feed [10]. The reason for the switch from $\mathrm{EtOH}$ to $\mathrm{MeOH}$ was simply the fact that data of the performance in EtOH were not available for the traditionally prepared membranes. Jimenez Solomon et al. did work with $\mathrm{MeOH}$ as a feed solution, which is why we measured the performance of our membranes in $\mathrm{MeOH}$ (and were able to make a comparison). The $\mathrm{MeOH}$ permeance of the SIM membrane was almost three times as high as that of the traditionally 
prepared membrane $\left(4.4 \mathrm{~L} / \mathrm{m}^{2} \mathrm{~h}\right.$ bar and $1.5 \mathrm{~L} / \mathrm{m}^{2} \mathrm{~h}$ bar, respectively). However, since the RB retention of the TFC SIM membrane was 93\%, while the molecular weight cut-off of the traditionally prepared membrane was below $400 \mathrm{Da}$, the latter is likely to have a more dense top-layer structure. The trade-off between permeance and selectivity is a well-known issue in membrane technology. Depending on the specific requirements, the most suitable membrane should be selected for each application. We would like to emphasize that a different synthesis method will inherently produce membranes with a different structure, hence, different performance, making it somewhat uncertain to accurately compare the performance of membranes prepared via different synthesis methods. The true comparison of both methods would only be possible after having optimized all synthesis parameters involved towards a certain application. This is however out of the scope of this work, which offers a 'proof of principle' only for the new simplified synthesis method.

The mass intensity of the SIM process was compared with the mass intensity of the traditional process to support the actual "green" improvement in an objective, quantitative manner. The definition of mass intensity was originally proposed excluding water, but the use of water has recently become incorporated more frequently [15]. Since water is used for washing steps, but also as a reaction medium in these synthesis processes, its impact is significant and both values are presented in Table 3 (detailed calculations can be found in the supplementary material).

Table 3. Mass and solvent intensity of the SRNF TFC membrane synthesis process via the SIM method, proposed in this work, and via the traditional method, as proposed by Jimenez Solomon et al. [10, 11].

\begin{tabular}{lccc}
\hline & including water & SIM & Traditional \\
\hline \multirow{2}{*}{ Mass intensity } & yes & 272 & 565 \\
& no & 85 & 253 \\
Solvent intensity & yes & 269 & 519 \\
& no & 82 & 208 \\
\hline
\end{tabular}

The mass intensity of the traditional process is reduced by $52 \%$ in the SIM process when including water, and by $66 \%$ when excluding water. Since the SIM process no longer requires the use of IPA during the membrane synthesis, the solvent intensity of both processes was determined as well (Table 3 ). The value of the traditional process is again reduced by $48 \%$ by 
the SIM process when water is included, and by $61 \%$ when water is excluded from the calculations. These values clearly indicate that the SIM process effectively reduces the amount of material needed to synthesize a SRNF TFC membrane and consequently creates less waste during the membrane synthesis.

\section{CONCLUSION}

It was demonstrated that the synthesis process of TFC membranes can be greatly simplified and optimized by adding amines already to the coagulation bath of the support without compromising on membrane performance. This new method eliminates the need for membrane conditioning (using e.g. polyethylene glycol and large quantities of IPA) in comparison with the traditionally used process, hence, reduces the amount of chemicals and eliminates the creation of waste streams during the membrane synthesis. Besides materials, also time and the number of manipulations are reduced during the production process.

The performance of several amines as crosslinker and monomer was tested and the use of a mixture consisting of HDA (as a crosslinker) and MPD (as a monomer for PA top-layer formation) in the coagulation bath was found to be optimal. Solvent activation with DMF was shown to have a major impact on the TFC membrane performance: both permeance and RB retention increased after storage in DMF.

In conclusion, the newly developed SIM method successfully combines multiple synthesis steps into one, minimizes the use of materials and makes the SRNF TFC synthesis process significantly greener, faster and more efficient. Moreover, membranes with excellent combinations of retentions and permeances can be obtained this way. After further optimization, it is possible that these TFC membranes, and the corresponding SIM method, can play an important role in the industrial purification of solvents.

\section{ACKNOWLEDGEMENTS}

The authors are grateful for the financial support from the OT $(11 / 061)$ funding from KU Leuven; the I.A.P - P.A.I. grant (IAP 7/05 FS2) from the Belgian Federal Government and the long term Methusalem (CASAS) funding by the Flemish Government. Also, we wish to thank the Laboratory for Entomology of KU Leuven for chemicals and procedures for TEM. 
E.D. acknowledges the Agency for Innovation by Science and Technology (IWTVlaanderen) for financial support.

6. APPENDIX A. SUPPLEMENTARY MATERIAL: Calculation of Mass intensity and Solvent intensity for both traditional and SIM method to prepare SRNF TFC (PA on crosslinked PI) membranes (Table I) and retention data of the smaller dyes methylene blue and methyl orange (Table II).

\section{REFERENCES}

[1] W.J. Lau, A.F. Ismail, N. Misdan, M.A. Kassim, A recent progress in thin film composite membrane: A review, Desalination, 287 (2012) 190-199.

[2] A. Cano-Odena, P. Vandezande, D. Fournier, W. Van Camp, F.E. Du Prez, I.F.J. Vankelecom, Solvent-Resistant Nanofiltration for Product Purification and Catalyst Recovery in Click Chemistry Reactions, Chem. Eur. J., 16 (2010) 1061-1067.

[3] L.S. White, Development of large-scale applications in organic solvent nanofiltration and pervaporation for chemical and refining processes, J. Membr. Sci., 286 (2006) 26-35.

[4] E.M. Rundquist, C.J. Pink, A.G. Livingston, Organic solvent nanofiltration: a potential alternative to distillation for solvent recovery from crystallisation mother liquors, Green Chem., 14 (2012) 2197 2205.

[5] P. Vandezande, L.E.M. Gevers, I.F.J. Vankelecom, Solvent resistant nanofiltration: separating on a molecular level, Chem. Soc. Rev., 37 (2008) 365-405.

[6] R.J. Petersen, Composite reverse osmosis and nanofiltration membranes, J. Membr. Sci., 83 (1993) 81-150.

[7] S. Hermans, H. Mariën, E. Dom, R. Bernstein, I.F.J. Vankelecom, Simplified synthesis route for interfacially polymerized polyamide membranes, J. Membr. Sci., 451 (2014) 148-156.

[8] K. Vanherck, P. Vandezande, S.O. Aldea, I.F.J. Vankelecom, Cross-linked polyimide membranes for solvent resistant nanofiltration in aprotic solvents, J. Membr. Sci., 320 (2008) 468-476.

[9] K. Vanherck, G. Koeckelberghs, I.F.J. Vankelecom, Crosslinking polyimides for membrane applications: A review, Prog. Polym. Sci., 38 (2013) 874-896.

[10] M.F. Jimenez Solomon, Y. Bhole, A.G. Livingston, High flux membranes for organic solvent nanofiltration (OSN) - Interfacial polymerization with solvent activation, J. Membr. Sci., 423-424 (2012) 371-382.

[11] M.F. Jimenez Solomon, Y. Bhole, A.G. Livingston, High flux hydrophobic membranes for organic solvent nanofiltration (OSN) - Interfacial polymerization, surface modification and solvent activation, J. Membr. Sci., 434 (2013) 193-203.

[12] K. Vanherck, A. Cano-Odena, G. Koeckelberghs, T. Dedroog, I. Vankelecom, A simplified diamine crosslinking method for PI nanofiltration membranes, J. Membr. Sci., 353 (2010) 135-143. 
[13] P. Vandezande, L.E.M. Gevers, J.S. Paul, I.F.J. Vankelecom, P.A. Jacobs, High throughput screening for rapid development of membranes and membrane processes, J. Membr. Sci., 250 (2005) 305-310.

[14] High Throughput Membrane Systems Leuven, www.html-membrane.be (2014).

[15] C. Jimenez-Gonzalez, D.J.C. Constable, C.S. Ponder, Evaluating the "Greenness" of chemical processes and products in the pharmaceutical industry-a green metrics primer, Chem. Soc. Rev., 41 (2012) 1485-1498.

[16] C.E. Powell, X.J. Duthie, S.E. Kentish, G.G. Qiao, G.W. Stevens, Reversible diamine crosslinking of polyimide membranes, J. Membr. Sci., 291 (2007) 199-209.

[17] Y. Liu, R. Wang, T.-S. Chung, Chemical cross-linking modification of polyimide membranes for gas separation, J. Membr. Sci., 189 (2001) 231-239.

[18] I. Soroko, Y. Bhole, A.G. Livingston, Environmentally friendly route for the preparation of solvent resistant polyimide nanofiltration membranes, Green Chem., 13 (2011) 162-168.

[19] Y.H. See Toh, F.W. Lim, A.G. Livingston, Polymeric membranes for nanofiltration in polar aprotic solvents, J. Membr. Sci., 301 (2007) 3-10.

[20] P. Vandezande, K. Vanherck, I.F.J. Vankelecom, Cross-linked polyimide membranes, European Patent 2,481,475 (2012).

[21] M.-J. Han, S.-T. Nam, Thermodynamic and rheological variation in polysulfone solution by PVP and its effect in the preparation of phase inversion membrane, J. Membr. Sci., 202 (2002) 55-61.

[22] W.M. Meylan, P.H. Howard, Atom/fragment contribution method for estimating octanol-water partition coefficients, J. Pharm. Sci., 84 (1995) 83-92.

[23] L. Shao, L. Liu, S.-X. Cheng, Y.-D. Huang, J. Ma, Comparison of diamino cross-linking in different polyimide solutions and membranes by precipitation observation and gas transport, J. Membr. Sci., 312 (2008) 174-185.

[24] A.K. Ghosh, B.-H. Jeong, X. Huang, E.M.V. Hoek, Impacts of reaction and curing conditions on polyamide composite reverse osmosis membrane properties, J. Membr. Sci., 311 (2008) 34-45.

[25] S.-H. Huang, C.-J. Hsu, D.-J. Liaw, C.-C. Hu, K.-R. Lee, J.-Y. Lai, Effect of chemical structures of amines on physicochemical properties of active layers and dehydration of isopropanol through interfacially polymerized thin-film composite membranes, J. Membr. Sci., 307 (2008) 73-81.

[26] I.J. Roh, Influence of rupture strength of interfacially polymerized thin-film structure on the performance of polyamide composite membranes, J. Membr. Sci., 198 (2002) 63-74.

[27] S.M. Aharoni, The solubility parameters of aromatic polyamides, J. Appl. Polym. Sci., 45 (1992) 813-817.

[28] P. Gorgojo, M.F. Jimenez-Solomon, A.G. Livingston, Polyamide thin film composite membranes on cross-linked polyimide supports: Improvement of RO performance via activating solvent, Desalination, 344 (2014) 181-188.

[29] A. Kulkarni, D. Mukherjee, W.N. Gill, Flux enhancement by hydrophilization of thin film composite reverse osmosis membranes, J. Membr. Sci., 114 (1996) 39-50. 
[30] D. Mukherjee, A. Kulkarni, W.N. Gill, Chemical treatment for improved performance of reverse osmosis membranes, Desalination, 104 (1996) 239-249.

[31] M.E. Rezac, J.D. Le Roux, H. Chen, D.R. Paul, W.J. Koros, Effect of mild solvent posttreatments on the gas transport properties of glassy polymer membranes, J. Membr. Sci., 90 (1994) 213-229. 


\section{Supplementary Information}

Table I:

Calculation of Mass intensity and Solvent intensity for both traditional and SIM method to prepare SRNF TFC (PA on crosslinked PI) membranes.

area of A4 size membrane sheet $(21 \mathrm{~cm} \mathrm{x} 29.7 \mathrm{~cm})$ is $623.7 \mathrm{~cm}^{2}$

assume one bath is $1 \mathrm{~cm}$ of liquid in height, hence $623.7 \mathrm{~cm}^{3}$

density IPA

$0.786 \mathrm{~g} / \mathrm{cm}^{3}$

density PEG $400 \quad 1.126 \mathrm{~g} / \mathrm{cm}^{3}$

density hexane $\quad 0.655 \mathrm{~g} / \mathrm{cm}^{3}$

\section{Traditional method}

(Jimenez-Solomon et al. $)^{10,11}$

substance

\section{SIM method}

amount

unit substance

24\%(w/w) P84 PI in DMSO (dope solution)

15 min immersion in water bath

dope solution

$60 \mathrm{~min}$ in fresh water bath

$624 \mathrm{~g}$ water

$14 \%$ (w/w) Matrimid PI in NMP/THF (3:1) (dope solution)

$30 \mathrm{~min}$ immersion in $0.5 \%(\mathrm{w} / \mathrm{v}) \mathrm{HDA}$ and $2 \%(\mathrm{w} / \mathrm{v}) \mathrm{MPD}$

$20 \mathrm{~g}$ dope solution

$624 \mathrm{~g}$ wate

IPA solvent exchange bath

IPA

water

16h in IPA/HDA, HDA concentration

unknown $(1.0 \text { or } 2.5 \mathrm{wt} \% \text { in Soroko et al. })^{14}$

assume $0.5 \%(\mathrm{w} / \mathrm{v}) \mathrm{HDA}$

$3 \mathrm{~h}$ washed with IPA

overnight in PEG 400/IPA (3:2 volume)

$490 \mathrm{~g} \quad$ IPA

$3 \mathrm{~g}$ HDA

$490 \mathrm{~g}$ IPA

$196 \mathrm{~g}$ IPA

$421 \mathrm{~g}$ PEG 400

wiped with tissue and dried

2 min placed in aqueous $2 \%(\mathrm{w} / \mathrm{v}) \mathrm{MPD}$

solution

$624 \mathrm{~g}$ wate

$12 \mathrm{~g}$ MPD

wiper

$1 \mathrm{~min} 0.1 \%$ (w/v) TMC in hexane

$\begin{array}{rll}408 & \mathrm{~g} & \text { hexane } \\ 0.6 & \mathrm{~g} & \text { TMC } \\ 408 & \mathrm{~g} & \text { hexane } \\ & & \\ 624 & \mathrm{~g} & \text { water } \\ 624 & \mathrm{~g} & \text { water }\end{array}$

$1 \mathrm{~min}$ immersed in a $0.1 \%(\mathrm{w} / \mathrm{v}) \mathrm{TMC}$ in

hexane

rinsed with water

stored in water of $4^{\circ} \mathrm{C}$
$408 \mathrm{~g}$ hexane

$0.6 \mathrm{~g}$ TMC

$624 \mathrm{~g}$ water

$624 \mathrm{~g}$ water

rinse with hexane

1 min air drying

rinsed with water

stored in water 


\begin{tabular}{|c|c|c|c|c|c|}
\hline mass of all materials used & $5651 \mathrm{~g}$ & & mass of all materials used & 2724 & $\mathrm{~g}$ \\
\hline mass of water used & $3119 \mathrm{~g}$ & & mass of water used & 1871 & $\mathrm{~g}$ \\
\hline mass of all materials used excluding water & $2533 \mathrm{~g}$ & & mass of all materials used excluding water & 853 & $\mathrm{~g}$ \\
\hline mass of all solvents used with water & $5194 \mathrm{~g}$ & & mass of all solvents used with water & 2688 & $\mathrm{~g}$ \\
\hline mass of all solvents used excluding water & $2075 \mathrm{~g}$ & & mass of all solvents used excluding water & 817 & $\mathrm{~g}$ \\
\hline mass of product (one A4 sheet, with nonwoven) & $10 \mathrm{~g}$ & & mass of product (one A4 sheet, with nonwoven) & 10 & $\mathrm{~g}$ \\
\hline Mass intensity (with water) & 565 & & Mass intensity (with water) & 272 & \\
\hline Mass intensity (excluding water) & 253 & & Mass intensity (excluding water) & 85 & \\
\hline \multirow[t]{2}{*}{ ratio Mass intensity traditional / SIM } & \multirow{2}{*}{$\begin{array}{l}\text { with water } \\
\text { excluding water }\end{array}$} & 2.1 & & & \\
\hline & & 3.0 & & & \\
\hline Solvent intensity (with water) & 519 & \multicolumn{2}{|r|}{ Solvent intensity (with water) } & 269 & \\
\hline Solvent intensity (excluding water) & 208 & & Solvent intensity (excluding water) & 82 & \\
\hline \multirow[t]{2}{*}{ ratio Solvent intensity traditional / SIM } & \multirow{2}{*}{$\begin{array}{l}\text { with water } \\
\text { excluding water }\end{array}$} & 1.9 & & & \\
\hline & & 2.5 & & & \\
\hline
\end{tabular}


Additional filtrations of the HDA/MPD membranes were done with methylene blue (MB) and methyl orange (MO) as test solutes in an EtOH feed. These dyes have a MW of 374 and $327 \mathrm{~g} / \mathrm{mol}$, respectively, which is lower than that of RB (1017 g/mol). To simultaneously test the effect of the solute charge on the retention, two dyes with a similar size but opposite charge were used (positive for MB, negative for MO). Solute characteristics and chemical structures are shown in Table II.

Table II: Chemical structure, molecular size and charge of the applied dyes.

Solute Size (g/mol) Charge

The obtained retentions of the TFC membrane for the newly investigated dyes MB and MO were $15 \%$ and $62 \%$, respectively. The membrane showed the highest retention for the largest solute (RB), but in addition, a clear difference between the retention of MB and MO was observable. This could be due to the negatively charged surface of the PA top layer, caused by the presence of carboxylic acids (originating from hydrolysis of acyl chlorides) ${ }^{\mathrm{a}}$. The positively charged MB can be attracted by these groups. After filtration, these membranes kept a dark blue color, clearly indicating the high $\mathrm{MB}$ concentration on the membrane. The higher the dye concentration at the membrane surface, the higher the amount of dye that will diffuse through the membrane, causing a lower retention. Since MO is negatively charged, it will rather be repelled by the carboxylic groups in the top layer, hindering sorption on the membrane. Despite the similar MW of MO and MB, the TFC membrane exhibited a higher retention for MO than for MB. Besides the molecular size, the solute charge thus also plays 
a role in the retention mechanism of the TFC membrane in organic solvents (similar to the well-known Donnan ion exclusion mechanism in aqueous applications) $)^{\text {b,c }}$.

${ }^{a}$ W.J. Lau, A.F. Ismail, N. Misdan, M.A. Kassim, A recent progress in thin film composite membrane: A review, Desalination, 287 (2012) 190-199.

${ }^{b}$ R.J. Petersen, Composite reverse osmosis and nanofiltration membranes, J. Membr. Sci., 83 (1993) 81-150.

${ }^{c}$ S. Bason, Y. Oren, V. Freger, Characterization of ion transport in thin films using electrochemical impedance spectroscopy: II: Examination of the polyamide layer of RO membranes, J. Membr. Sci., 302 (2007) 10-19. 